\title{
A Matrix Inequality for the Inversions of the Restrictions of a Positive Definite Hermitian Matrix
}

\author{
Weixiong Mai ${ }^{1}$, Mo Yan ${ }^{1}$, Tao Qian ${ }^{1}$, Matteo Dalla Riva ${ }^{2}$, Saburou Saitoh ${ }^{2}$ \\ ${ }^{1}$ Department of Mathematics, Faculty of Science and Technology, University of Macau, \\ Macau, China \\ ${ }^{2}$ Center for Research and Development in Mathematics and Applications, \\ Department of Mathematics, University of Aveiro, Aveiro, Portugal \\ Email: saburou.saitoh@gmail.com
}

Received October 17, 2013; revised November 15, 2013; accepted November 22, 2013

Copyright (C) 2013 Weixiong Mai et al. This is an open access article distributed under the Creative Commons Attribution License, which permits unrestricted use, distribution, and reproduction in any medium, provided the original work is properly cited.

\begin{abstract}
We exploit the theory of reproducing kernels to deduce a matrix inequality for the inverse of the restriction of a positive definite Hermitian matrix.

Keywords: Reproducing Kernel; Positive Definite Hermitian Matrix; Quadratic Inequality; Inversion of Positive Definite Hermitian Matrix; Restriction of Positive Definite Hermitian Matrix; Schur Complement; Block Matrix
\end{abstract}

\section{Introduction and Results}

By exploiting the general structure of reproducing kernel Hilbert spaces, it is possible to prove very interesting norm inequalities (see, e.g., [1,2]). A typical result is as follows.
Let $D$ be an $N$-ply connected regular domain whose boundary consists of disjoint analytic Jordan curves. Let $\varphi, \psi \in H_{2}(D)$ be analytic Hardy functions with index two. Then the following generalised isoperimetric inequality holds,

$$
\frac{1}{\pi} \iint_{D}|\varphi(z) \psi(z)|^{2} \mathrm{~d} x \mathrm{~d} y \leq \frac{1}{2 \pi} \int_{\partial D}|\varphi(z)|^{2}|\mathrm{~d} z| \frac{1}{2 \pi} \int_{\partial D}|\psi(z)|^{2}|\mathrm{~d} z|, \quad z=x+i y
$$

Moreover, we can completely describe the cases for which we have the equality instead of the inequality here above. Without the theory of reproducing kernels, such a simple and beautiful inequality could not be derived (see $[2,3]$ for the details).

In this paper we introduce a new inequality. Let $A_{n}=\left\{a_{i, j}\right\}_{i, j=1}^{n}$ be a positive definite Hermitian matrix. Let $0<m<n$ and let $A_{m}=\left\{a_{i, j}\right\}_{i, j=1}^{m}$ be the restriction of $A_{m}$ to an $m$ dimensional subspace of $\mathbb{C}^{n}$. Without loss of generality, assume that $A_{m}$ is the $m \times m$ leading principal minor of $A_{n}$. Let $A_{n}^{-1}$ and $A_{m}^{-1}$ denote the inverse of $A_{n}$ and of $A_{m}$, respectively. Then we have the following results.

Theorem 1.1 If $x \in\left(x_{1}, \cdots, x_{n}\right) \in \mathbb{C}^{n}$ and $x_{(m)}$ is the vector of $\mathbb{C}^{m}$ defined by $x_{(m)} \equiv\left(x_{1}, \cdots, x_{m}\right)$, then

$$
x_{(m)}^{*} A_{m}^{-1} X_{(m)} \leq x^{*} A_{n}^{-1} x .
$$

Here $^{*}$ denotes conjugate transpose. As an immediate consequence, one also obtains the following corollary.

Corollary 1.2 If $\left(A_{n}^{-1}\right)_{m}$ is the restriction of the matrix $A_{n}^{-1}$ to $\mathbb{C}^{m}$, then

$$
A_{m}^{-1} \leq\left(A_{n}^{-1}\right)_{m} .
$$

Here $\leq$ denotes the positive definite order, i.e., if $M$ and $N$ are square matrices, we say that $M \leq N$ if $N-M$ is a positive semi-definite matrix.

We observe that for $n=2$, such results can be checked directly. However, for $n \geq 3$, the result of Theorem 1.1 is not intuitive and appears mysterious, at least at first glance.

\section{Proof of the Results}

The proof of Theorem 1.1 is based on the theory of 
reproducing kernels. Therefore, we begin by introducing some notions and results which are used in the sequel.

\subsection{Reproducing Kernels}

Let $E$ be an arbitrary abstract (non-void) set. Let $\mathscr{T}(E)$ denote the set of all complex-valued functions on $E$. A reproducing kernel Hilbert spaces (RKHS for short) on the set $E$ is a Hilbert space $\mathscr{K} \subset \mathscr{F}(E)$ endowed with a function $K: E \times E \rightarrow \mathscr{C}$, which is called the reproducing kernel and which satisfies the reproducing property. Namely we have

$$
K_{p} \equiv K(\cdot, p) \in \mathscr{C} \text { for all } p \in E
$$

and

$$
f(p)=\left\langle f, K_{p}\right\rangle_{\mathscr{R}}
$$

for all $p \in E$ and for all $f \in \mathscr{C}$. We denote by $H_{K}(E)$ (or $H_{K}$ ) the reproducing kernel Hilbert space $\mathscr{H}$ whose corresponding reproducing function is $K$.

A complex-valued function $K: E \times E \rightarrow \mathbb{C}$ is called a positive definite quadratic form function on the set $E$, or shortly, positive definite function, if, for an arbitrary function $X: E \rightarrow \mathbb{C}$ and for any finite subset $F$ of $E$, one has

$$
\sum_{p, q \in F} \overline{X(p)} X(q) K(p, q) \geq 0 .
$$

By a fundamental theorem, we know that, for any positive definite quadratic form function $K$ on $E$, there exists a unique reproducing kernel Hilbert space on $E$ with reproducing kernel $K$. So, in a sense, the correspondence between the reproducing kernel $K$ and the reproducing kernel Hilbert space $H_{K}(E)$ is one to one.

A simple example of positive definite quadratic form function is a positive definite Hermitian matrix.

Example 2.1 Let $E=\left\{p_{1}, p_{2}, \cdots, p_{n}\right\}$ be a set consisting of $n$ distinct points. Let $A_{n} \equiv\left\{a_{i j}\right\}_{i, j=1}^{n}$ be $a$ strictly positive $n \times n$ Hermitian matrix. Let $A_{n}^{-1} \equiv\left\{b_{i j}\right\}_{i, j=1}^{n}$ denote the inverse of $A_{n}$. Then the space

$\mathcal{F}(E)$ of the complex valued functions on $E$, endowed with the inner product

$$
\begin{aligned}
\langle f, g\rangle_{H_{\bar{A}_{n}}(E)} & \equiv \sum_{i, j=1}^{n} f\left(x_{i}\right) b_{i j} \overline{g\left(p_{j}\right)} \\
& =\overline{\left(g\left(p_{1}\right), g\left(p_{2}\right), \cdots, g\left(p_{n}\right)\right)} A_{n}^{-1}\left(\begin{array}{c}
f\left(p_{1}\right) \\
f\left(p_{2}\right) \\
\vdots \\
f\left(p_{n}\right)
\end{array}\right),
\end{aligned}
$$

is a reproducing kernel Hilbert (complex Euclidean) space with reproducing kernel $K$ defined by $K\left(p_{i}, p_{j}\right)=a_{j i}$ for all $i, j=1, \cdots, n$.

Indeed, the validity of (3) follows by a straightforward calculation. To prove (4) we observe that

$$
\begin{aligned}
\left\langle f, K\left(\cdot, p_{k}\right)\right\rangle_{H_{K_{\bar{A}_{n}}}(E)} & =\sum_{i, j=1}^{n} f\left(p_{i}\right) b_{i j} \overline{K\left(p_{j}, p_{k}\right)} \\
& =\sum_{i, j=1}^{n} f\left(p_{i}\right) b_{i j} a_{j k}=f\left(p_{k}\right)
\end{aligned}
$$

for all $k=1, \cdots, n$ (note that $\overline{a_{k j}}=a_{j k}$ ). Thus $H_{\bar{A}_{n}}(E)$ coincides with the reproducing kernel Hilbert space $H_{K}(E)$. In particular the norm induced by the product $\langle\cdot, \cdot\rangle_{H_{\bar{A}_{n}}(E)}$ coincides with the norm of $H_{K}(E)$.

We ${ }^{-}$can thus combine the two theories of postitive definite Hermitian matrices and of reproducing kernels (see [4-12]).

\subsection{Restriction of a Reproducing Kernel}

The validity of Theorem 1.1 follows by the properties of the restriction of a reproducing kernel in a general setting. Let $E$ be a non-empty set and let $E_{0}$ be a non-empty subset of $E$. Let $K: E \times E \rightarrow \mathbb{C}$ be a positive definite quadratic form function. Then the restriction $\left.K\right|_{E_{0} \times E_{0}}$ of $K$ to $E_{0} \times E_{0}$ is a positive definite quadratic form function on $E_{0} \times E_{0}$ and the relation between $H_{K}(E)$ and $H_{\left.K\right|_{E_{0} \times E_{0}}}\left(E_{0}\right)$ is given by the following statement.

Proposition 2.1 (Restriction of RKHS) Let $E$ be a non-empty set and let $E_{0}$ be a non-empty subset of $E$. Let $K: E \times E \rightarrow \mathbb{C}$ be a positive definite quadratic form function. Then the Hilbert space defined by the positive definite quadratic form function $\left.K\right|_{E_{0} \times E_{0}}$ is given by

$$
H_{K_{E_{0} \times E_{0}}}\left(E_{0}\right)=\left\{f \in \mathscr{T}\left(E_{0}\right): f=\left.\tilde{f}\right|_{E_{0}} \text { for some } \tilde{f} \in H_{K}(E)\right\} \text {. }
$$

Furthermore, the norm of $H_{\left.K\right|_{E_{0} \times E_{0}}}\left(E_{0}\right)$ is expressed in terms of the norm of $H_{K}(E)$ by the following equality,

$$
\|f\|_{H_{K_{E_{0} \times E_{0}}}\left(E_{0}\right)}=\min \left\{\|\tilde{f}\|_{H_{K}(E)}: \tilde{f} \in H_{K}(E), f=\left.\tilde{f}\right|_{E_{0}}\right\}
$$

which holds for all $f \in H_{\left.K\right|_{E_{0} \times E_{0}}}\left(E_{0}\right)$.

See [1] for the details.

\subsection{Proof of Theorem 1.1}

Let $n, m \in \mathbb{N}$ with $0<m<n$. Let $E \equiv\left\{p_{1}, p_{2}, \cdots, p_{n}\right\}$ and $E_{0} \equiv\left\{p_{1}, p_{2}, \cdots, p_{m}\right\}$. Let $K$ be the positive 
definite quadratic form function on $E$ defined by $K\left(p_{i}, p_{j}\right)=a_{j i}$ for all $i, j=1, \cdots, n$. Let $x \equiv\left(x_{1}, \cdots, x_{n}\right) \in \mathbb{C}^{n}$ and $x_{(m)} \equiv\left(x_{1}, \cdots, x_{m}\right) \in \mathbb{C}^{m}$. Let $\tilde{f}$ be the function on $E$ defined by $\tilde{f}\left(p_{i}\right)=x_{i}$ for all $i=1, \cdots, n$. Let $\left.f \equiv \tilde{f}\right|_{E_{0}}$. Then we have

$\|\tilde{f}\|_{H_{K}(E)}^{2}=\langle f, f\rangle_{H_{K}(E)}=x^{*} A_{n}^{-1} x$ and

$\|f\|_{H_{K_{E_{0} \times E_{0}}}\left(E_{0}\right)}^{2}=\langle f, f\rangle_{H_{\left.K\right|_{E_{0} \times E_{0}}}\left(E_{0}\right)}=x_{(m)}^{*} A_{m}^{-1} x_{(m)}$. Thus

implies that $x_{(m)}^{*} A_{m}^{-1} x_{(m)} \leq x^{*} A_{n}^{-1} x$.

\section{An Alternative Proof Based on Schur Complement}

We provide in this section a direct proof of Theorem 1.1 based on the properties of the Schur complement (cf., e.g., [13]). Let $n, m \in \mathbb{N}$ with $0<m<n$. Let $A_{n}$ be a positive definite Hermitian $n \times n$ matrix and assume that

$$
A_{n} \equiv\left(\begin{array}{cc}
A_{m} & B \\
B^{*} & A_{n-m}
\end{array}\right)
$$

where $A_{m}$ is an $m \times m$ matrix, $A_{n-m}$ is an $(n-m) \times(n-m)$ matrix, and $B$ is an $(n-m) \times m$ matrix. Observe that $A_{m}$ is positive definite and henceforth invertible. Then the inverse $A_{n}$ can be written in the form

$$
A_{n}^{-1} \equiv\left(\begin{array}{cc}
A_{m}^{-1}+A_{m}^{-1} B S_{n-m}^{-1} B^{*} A_{m}^{-1} & -A_{m}^{-1} B S_{n-m}^{-1} \\
-S_{n-m}^{-1} B^{*} A_{m}^{-1} & S_{n-m}^{-1}
\end{array}\right)
$$

where $S_{n-m} \equiv A_{n-m}-B^{*} A_{m}^{-1} B$ is the Schur complement with respect to $A_{m}$. Since $A_{n}>0$ we also have $A_{n}^{-1}>0$ which implies that $S_{n-m}^{-1}>0$. We now observe that the validity of Theorem 1.1 is equivalent to say that the matrix $M$ defined by

$$
M \equiv A_{n}^{-1}-\left(\begin{array}{cc}
A_{m}^{-1} & 0 \\
0 & 0
\end{array}\right)=\left(\begin{array}{cc}
A_{m}^{-1} B S_{n-m}^{-1} B^{*} A_{m}^{-1} & -A_{m}^{-1} B S_{n-m}^{-1} \\
-S_{n-m}^{-1} B^{*} A_{m}^{-1} & S_{n-m}^{-1}
\end{array}\right)
$$

is positive semi-definite. Let $x \in \mathbb{C}^{m}$ and $y \in \mathbb{C}^{n-m}$. Then we calculate

$$
\begin{aligned}
& (x, y)^{*} M(x, y) \\
= & x^{*} A_{m}^{-1} B S_{n-m}^{-1} B^{*} A_{m}^{-1} x-y^{*} S_{n-m}^{-1} B^{*} A_{m}^{-1} x \\
& -x^{*} A_{m}^{-1} B S_{n-m}^{-1} y+y^{*} S_{n-m}^{-1} y \\
= & \left(B^{*} A_{m}^{-1} x\right)^{*} S_{n-m}^{-1}\left(B^{*} A_{m}^{-1} x\right)-y^{*} S_{n-m}^{-1}\left(B^{*} A_{m}^{-1} x\right) \\
& -\left(B^{*} A_{m}^{-1} x\right)^{*} S_{n-m}^{-1} y+y^{*} S_{n-m}^{-1} y \\
= & \left(B^{*} A_{m}^{-1} x, y\right)^{*}\left(\begin{array}{cc}
S_{n-m}^{-1} & -S_{n-m}^{-1} \\
-S_{n-m}^{-1} & S_{n-m}^{-1}
\end{array}\right)\left(B^{*} A_{m}^{-1} x, y\right)
\end{aligned}
$$

(here we understand that $(x, y)$ and $\left(B^{*} A_{m}^{-1} x, y\right)$ are column vectors). Now we observe that

$$
\left(\begin{array}{cc}
S_{n-m}^{-1} & -S_{n-m}^{-1} \\
-S_{n-m}^{-1} & S_{n-m}^{-1}
\end{array}\right)=\left(\begin{array}{cc}
1 & -1 \\
-1 & 1
\end{array}\right) \otimes S_{n-m}^{-1}
$$

where $\otimes$ denotes the Kronecker product of matrices. It is known that the Kronecker product of positive semidefinite matrices is positive semi-definite. Now

$$
\left(\begin{array}{cc}
1 & -1 \\
-1 & 1
\end{array}\right) \geq 0
$$

and $S_{n-m}^{-1}>0$, hence

$$
\left(\begin{array}{cc}
S_{n-m}^{-1} & -S_{n-m}^{-1} \\
-S_{n-m}^{-1} & S_{n-m}^{-1}
\end{array}\right)
$$

is positive semi-definite and accordingly $(x, y)^{*} M(x, y) \geq 0$. Our proof is completed.

\section{Remark}

The results in this paper were given implicitly in the extensive paper [14]. However, such results were not explicitly stated in the corresponding Theorem (Ultimate realization of reproducing kernel Hilbert spaces). For this reason, we wrote this paper where we clearly present our Theorem 1.1. We note that such ideas have arisen to our attention while analysing the structure of the theorem from the viewpoint of the support vector machine for the practical calculation.

\section{Acknowledgements}

The last author wishes to express his sincere gratitude to Professor Tsuyoshi Ando for providing exciting informations on system theory and the Schur complement. He is supported in part by the Grant-in-Aid for the Scientific Research (C)(2) (No. 24540113).

The research of M. Dalla Riva was supported by FEDER funds through COMPETE-Operational Programme Factors of Competitiveness ("Programa Operacional Factores de Competitividade") and by Portuguese funds through the Center for Research and Development in Mathematics and Applications (University of Aveiro) and the Portuguese Foundation for Science and Technology ("FCT_Fundação para a Ciência e a Tecnologia"), within project PEst-C/MAT/UI4106/2011 with the Compete number FCOMP-01-0124-FEDER-022690. The research was also supported by the Portuguese Foundation for Science and Technology ("FCT—Fundação para a Ciência e a Tecnologia") with the research grant SFRH/ BPD/64437/2009 and by "Progetto di Ateneo: Singular perturbation problems for differential operators"- University of Padova.

\section{REFERENCES}

[1] S. Saitoh, "Integral Transforms, Reproducing Kernels and 
Their Applications," Pitman Research Notes in Mathematics Series 369, Addison Wesley Longman, Harlow, 1997.

[2] S. Saitoh, "Theory of Reproducing Kernels: Applications to Approximate Solutions of Bounded Linear Operator Functions on Hilbert Spaces," American Mathematical Society Translations: Series 2, Vol. 230, American Mathematical Society, Providence, 2010.

[3] S. Saitoh, "The Bergman Norm and the Szegö Norm," Transactions of the American Mathematical Society, Vol. 249, No. 1-2, 1979, pp. 261-279.

[4] M. Asaduzzaman and S. Saitoh, "Inverses of a Family of Matrices and Generalizations of Pythagorean Theorem," Panamerican Mathematical Journal, Vol. 13, No. 4, 2003, pp. $45-53$.

[5] B. Mond, J. E. Pecaric and S. Saitoh, "History, Variations and Generalizations of an Inequality of Marcus," Riazi. The Journal of Karachi Mathematical Association, Vol. 16, No. 1, 1994, pp. 7-15.

[6] S. Saitoh, "Positive Definite Hermitian Matrices and Reproducing Kernels," Linear Algebra and Its Applications, Vol. 48, No. 1, 1982, pp. 119-130.

[7] S. Saitoh, "Quadratic Inequalities Deduced from the Theory of Reproducing Kernels," Linear Algebra and Its Applications, Vol. 93, No. 1, 1987, pp. 171-178.

[8] S. Saitoh, "Quadratic Inequalities Associated with Integrals of Reproducing Kernels," Linear Algebra and Its Applications, Vol. 101, No. 2, 1988, pp. 269-280.
[9] S. Saitoh, "Generalizations of the Triangle Inequality," JIPAM-Journal of Inequalities in Pure and Applied Mathematics, Vol. 4, No. 3, 2003, Article 62.

[10] Y. Sawano, "Pasting Reproducing Kernel Hilbert Spaces," Jaen Journal on Approximation, Vol. 3, No. 1, 2011, pp. 135-141.

[11] A. Yamada, "Oppenheim's Inequality and RKHS," Mathematical Inequalities \& Applications, Vol. 15, No. 2, 2012, pp. 449-456.

[12] A. Yamada, "Inequalities for Gram Matrices and Their Applications to Reproducing Kernel Hilbert Spaces," Taiwanese Journal of Mathematics, Vol. 17, No. 2, 2013, pp. 427-430.

[13] D. Carlson, "What Are Schur Complements, Anyway?" Linear Algebra and Its Applications, Vol. 74, No. 1, 1986, pp. 257-275.

[14] L. P. Castro, H. Fujiwara, M. M. Rodrigues, S. Saitoh and V. K. Tuan, "Aveiro Discretization Method in Mathematics: A New Discretization Principle," Mathematics without Boundaries: Surveys in Pure Mathematics, Edited by Panos Pardalos and Themistocles M. Rassias (to appear). $52 \mathrm{p}$. 